\title{
Theoretical and experimental study of cylindrical shock and heterogeneous detonation waves
}

\author{
J. A. NICHOLLS, M. SICHEL, R. FRY AND D. R. GLASS \\ Aerospace Engineering, University of Michigan, Ann Arbor, Michigan, U.S.A.
}

(Received 5 September 1973)

\begin{abstract}
A simplified theory of blast initiation of detonations in clouds of fuel in gaseous or droplet form is developed and agrees with the experiments described below. The flow is at first dominated by the strong blast wave but transition from blast to detonation behavior occurs near a critical radius $r$. where the blast energy and the heat of combustion contained in $r<r$. are equal. The complex flow in this transition region cannot be determined analytically. In the simplified theory the details of the transition region are ignored but the flow is represented by the self-similar solution for a strong blast wave for $r<r *$ and by the self-similar detonation solution for $r>r$.

The development of a sectored shock tube to study cylindrical shock waves and two-phase detonations is described. Data are presented for shock waves as well as for blast initiated detonations of a monodisperse spray of $400 \mu$ kerosene droplets in air at standard conditions. Two regimes of propagation were established experimentally: (1) the subcritical energy regime, where decoupling of shock and reaction zone results in a strong blast wave type decay and, (2) the supercritical energy regime, where the initially overdriven cylindrical detonation decays, at some critical radius, to its Chapman-Jouguet state. Experimentally determined critical radii and steady-state detonation velocity agree very well with theoretical predictions. Detonation velocity was found to be constant at the plane $\mathrm{C}-\mathrm{J}$ value for radius greater than the critical radius.
\end{abstract}

\section{Introduction}

THIS STUDY is directed to the initiation of detonation in two-phase (liquid-gas) mixtures by blast waves. In such cases one would expect strong blast wave behavior to be observed near the origin of the blast. However, at sufficient distances away and provided detonation was actually achieved, one would expect a constant velocity Chapman-Jouguet detonation. Accordingly, the theoretical portion of this paper considers strong blast wave theory for the cases of planar, cylindrical, and spherical geometry. Similarity solutions are discussed and some results presented. Further, the similarity solution for a detonation wave is discussed. Of course, there is a transition region between the two limiting solutions, which, for simplification, is assumed to occur discontinuously at a certain critical radius. Thus a much simplified model of the entire process is adopted.

The second part of the study is devoted to the experimental achievement of blast initiated detonations and how these results compared with theoretical predictions. For these studies only the cylindrical geometry case was considered. Initial effort was directed at the production of cylindrical blast waves and a determination of the effective energy release driving these waves. In later studies the shock wave impacted a cloud of kerosene drops and air, wherein the cloud was formed by 77 needles, each disturbed at the Rayleigh frequency and producing drops of the same size. Attention was given to those conditions under which 
detonation was established, and the overall cloud behavior as it compared to the simplified theoretical treatment.

The theoretical and experimental aspects of the study are now described.

\section{Theoretical analysis}

A simplified analysis of the propagation of a blast wave through a gaseous or two-phase combustible mixture is developed below. The reviews of Lee [1], Chernyi et al. [2], and Korobeinikov [3] summarize recent work on blast propagation through combustible mixtures. In discussing the propagation of blast waves through combustible mixtures [4] it is useful to define a critical blast wave radius $r$ such that the energy of combustion contained within $r$ is equal to the blast wave energy $E_{0}$. If $Q$ is the combustion energy per unit mass of fuel oxidizer mixture, then

$$
r *=\left(\nu E_{0} / \sigma_{\nu} Q \rho_{1}\right)^{1 / \nu}
$$

where $\nu=1,2,3$ and $\sigma_{\nu}=2,2 \pi, 4 \pi$ for planar cylindrical and spherical waves. When the blast radius $r_{s} \ll r_{*}$, the blast energy $E_{0}$ is dominant and asymptotic analysis [5] shows that the flow then can be described by the self-similar strong blast wave solution of Sedov [6] and Taylor [7]. When $r_{s} \gg r_{*}$ combustion dominates and the blast wave decays to a constant velocity Chapman-Jouguet (C-J) detonation, provided combustion is instantaneous at the shock front. The flow behind the C-J wave also is self-similar [6]. In the transitional region $r_{s} \sim O\left(r_{*}\right)$ the flow is no longer self-similar and various numerical, perturbation, and approximation techniques used to deal with this region are described by Lee[1] and Korobeinikov [4].

The theory shows [4 and 5] that transition to a C-J detonation always occurs when the combustion energy is released instantaneously, i.e., when the reaction zone thickness, $\Delta$, behind the shock front is negligibly small. With finite $\Delta$, the one-dimensional analysis of Korobeinikov et al.[8] shows that the flame zone separates from the leading shock precluding the formation of a steady $\mathrm{C}-\mathrm{J}$ detonation. While this flame separation is observed when missiles are fired through combustible mixtures [9], detonation can be initiated with blast waves even though the reaction zone thickness, $\Delta$, has some finite value for all detonations [10]. The key to the conflict between the results of the one-dimensional theories and observation is that two-dimensional effects are present within the reaction zone of most self-sustained detonations [11]. A complete theory of blast initiation must take these effects into account. As a preliminary step in this direction Bach et al.[10] have introduced a phenomenological theory of initiation. They find that with a finite reaction zone thickness $\Delta$, transition to a $\mathrm{C}-\mathrm{J}$ detonation will only occur when the blast energy $E_{0}$ exceeds a certain critical initiation value in accord with experimental observation. In terms of a characteristic explosion length, $r_{0}$, defined by

$$
r_{0}=\left(\frac{E_{0}}{k_{\nu} \rho_{1} a_{1}^{2}}\right)^{1 / \nu}=\left(\frac{E_{0}}{k_{\nu} \gamma_{1} p_{1}}\right)^{1 / \nu}
$$

blast initiation of a C-J detonation occurs only when the ratio $\left(\Delta / r_{0}\right)$ is less than a certain critical value. Here $k_{\nu}=1,2 \pi, 4 \pi$, for $\nu=1,2,3$, and $a_{1}$ is the speed of 
sound in the unburned fuel oxidizer mixture. An interesting feature of the theory is the occurrence of stable sub-C-J waves when $\Delta / r_{0}$ is near the critical initiation value.

The theoretical developments described above require considerable computation, particularly in the transitional region, $r_{1} \sim O\left(r_{*}\right)$. The theory of initiation is, in any case, only in a preliminary form. There are, however, a number of practical problems where the main features of blast propagation through explosive mixtures are desired with minimum computational effort. The results of Bach et al. [10], Korobeinikov [4], and Chernyi et al. [2] show that the transition from blast to C-J wave behavior, if it takes place, occurs when $r_{s} \sim r_{*}$, for both discontinuous and finite thickness detonation fronts. For rapid estimation of propagation properties it is therefore proposed to use the self-similar strong blast wave theory for $r_{s}<r_{*}$ and the self-similar C-J wave solution for $r_{s}>r_{*}$.

Such an ultra simple or zeroth-order theory cannot describe the details of the transitional flow, nor can it be used to predict initiation energies. However, the experiments described below show that the blast- $\mathrm{C}-\mathrm{J}$ wave transition occurs very rapidly, and that the zeroth-order theory predicts the propagation of the wave front remarkably well. The use of $r$ as the dividing point between the blast and $\mathrm{C}-\mathrm{J}$ solutions fits the experimentally observed result that the transitional radius increases with increased blast energy $E_{0}$. Korobeinikov [4] shows that the distributions of velocity, pressure, and density correspond to those behind a strong blast wave when $r_{s} / r_{*} \ll 1$, and to those behind a $\mathrm{C}-\mathrm{J}$ detonation when $r_{s} / r_{*} \gg 1$. In the zeroth-order solution it is assumed that the blast wave property variations are valid for $r_{s}<r$. while the C-J property distributions are used for $r_{s}>r_{*}$. The validity of this feature of the zeroth-order solution, which facilitates the computation of impulse and other blast effects, remains to be tested experimentally.

The details of the self-similar solution for strong blast waves is described in great detail by Sedov [6]. The key assumptions are that the shock pressure ratio $\left(p_{2} / p_{1}\right) \gg 1$, that the release of explosive energy is instantaneous, and that the fluid is a perfect gas with constant specific heats. Then it can be shown that the shock radius $r_{s}$, and the velocity $v_{2}$, pressure $p_{2}$, and temperature $T_{2}$ immediately behind the shock front are given by

$$
\begin{aligned}
& r_{s}=\left(\frac{E_{0}}{\alpha \rho_{1}}\right)^{1 / \nu+2} t^{2 / \nu+2} \\
& v_{2}=\frac{4}{(\nu+2)(\gamma+1)}\left(\frac{E_{0}}{\alpha \rho_{1}}\right)^{1 / \nu+2} t^{-\nu / \nu+2} \\
& p_{2}=\frac{8 \rho_{1}}{(\nu+2)^{2}(\gamma+1)}\left(\frac{E_{0}}{\alpha \rho_{1}}\right)^{2 / 2+\nu} t^{-2 \nu / \nu+2} \\
& T_{2}=\frac{8(\gamma-1)}{(\nu+2)^{2}(\gamma+1)^{2}}\left(\frac{E_{0}}{\alpha \rho_{1}}\right)^{2 / 2+\nu} t^{-2 \nu / \nu+2}
\end{aligned}
$$

The parameter $\alpha$, which is related to energy conservation, is a function of $\gamma$ and the dimensional index $\nu$ and may be found in Ref. [6]. Analytical solutions for $r / r$, 
$v / v_{2}, p / p_{2}, T / T_{2}$ and $\rho / \rho_{2}$ between the shock front and explosion center are presented by Sedov [6] as functions of the dimensionless velocity $V=v(t / r)$ and can be readily used to find the properties of the flow behind the explosion front. The self-similar solution described above fails near explosion center, and as indicated by Brode $e t$ al.[12] can only provide a crude approximation, and, with recent advances in numerical computation more accurate theoretical solutions have become available. However, the simplicity of the "old" blast wave theory is in the spirit of the zeroth-order theory proposed here and the results agree quite well with the experiments described below.

The conservation equations describing the self-similar flow behind a $\mathrm{C}-\mathrm{J}$ detonation can be reduced to the single ordinary differential Eq. (5)

$$
\frac{d z}{d V}=\frac{z\left[2(V-1)^{2}+(\nu-1)\left(\gamma_{2}-1\right) V(V-1)-2 z\right]}{V\left[(V-1)^{2}-\nu z\right]}
$$

where the dimensionless velocity $V$ and the variable $z$ are defined by

$$
\begin{array}{ll}
v=\frac{r}{t} V(\lambda) ; & z=\gamma_{2} \frac{P(\lambda)}{R(\lambda)} \\
p=\rho_{1}\left(r^{2} / t^{2}\right) P(\lambda) ; & \rho=\rho_{1} R(\lambda)
\end{array}
$$

$\lambda$ is the similarity variable $\left(r / r_{s}\right)$, while $\gamma_{2}$ is the ratio of specific heats of the combustion products. The numerical integration of Eq. (4) is the key problem in computing the self-similar flow behind a C-J detonation. The variation of $V$ and $z$ with the dimensionless radius $\left(r / r_{s}\right)$ can then be found by integrating

$$
\frac{d(\ln \lambda)}{d V}=\frac{z-(V-1)^{2}}{V\left[(V-1)^{2}-\nu z\right]}
$$

The flow behind the detonation is assumed to be isentropic so that $z$ and $R$ are related by

$$
\frac{\lambda^{2} z}{R^{\left(\gamma_{2}-1\right)}}=\frac{z_{2}}{R_{2}^{\left(\gamma_{2}-1\right)}}
$$

The integration of Eq. (4) is started at the point $\left(z_{2}, V_{2}\right)$ corresponding to the C-J conditions immediately behind the detonation front. Hence $\left(z_{2}, V_{2}\right)$ must be on the sonic parabola $z=(1-V)^{2}$ in the $z-V$ plane (Fig. 1). Accurate computation of the properties immediately downstream of the detonation front requires the tedious determination of chemical equilibrium for each fuel-oxidizer mixture and has been carried out using the Gordon-McBride numerical code [13]. Once the propagation velocity $C$; Mach number $M_{D}$ and ratios of specific heats $\gamma_{1}$ and $\gamma_{2}$, and the molecular weight $\mu_{2}$ of the combustion products have been determined using [13] other detonation properties can be determined with remarkable accuracy from the perfect gas Hugoniot Equations applied across the wave with changes in gas properties taken into account. Thus for $\mathrm{C}-\mathrm{J}$ detonations

where $z_{1}=M_{D}^{-2}$.

$$
\begin{aligned}
& R_{2}=\left[\frac{\gamma_{2}}{\gamma_{2}+1}\left(1+\frac{z_{1}}{\gamma_{1}}\right)\right]^{-1} \\
& z_{2}=\frac{\gamma_{2}^{2}}{\left(\gamma_{2}+1\right)^{2}}\left(1+\frac{z_{1}}{\gamma_{1}}\right)^{2}
\end{aligned}
$$






Fig. 1. The $V-z$ plane for a C-J detonation.

The detonation front is followed by an isentropic expansion through which the velocity of the combustion products drops to zero at the boundary of a stationary core region, which corresponds to the point $A: z=1, V=0$ in the $z-V$ plane. Point $A$ is a node and in the physical plane corresponds the characteristic separating the expansion wave from the stationary core. The stationary core is represented by the line $V=0$ extending from $z=1$ to $z=\infty$ which corresponds to the detonation center $r=0$. Some typical $z-V$ solution curves are shown in Fig. 1 for $\nu=1,2,3$. The $\nu=1$ solution is singular and coincides with the sonic parabola $z=$ $(1-V)^{2}$. Typical pressure profiles behind a stoichiometric $\mathrm{CH}_{4}$-air detonation computed as described here are shown in Fig. 2.

In computing $r *$ it is important to recognize that $Q$ is not the fuel heating value per unit mass of the fuel oxidizer mixture, for with dissociation combustion is rarely complete, particularly near stoichiometric conditions. However, from the perfect gas $\mathbf{C}-\mathrm{J}$ conditions it can be shown that

$$
Q=\frac{C^{2}}{2\left(\gamma_{2}^{2}-1\right)}=\frac{a_{1}^{2} M_{D}^{2}}{2\left(\gamma_{2}^{2}-1\right)}
$$

where now $C, \gamma_{2}$, and $M_{D}$ are taken from the exact computation[13] of the C-J conditions. With Eq. (9) the expression for $r$. can be written

$$
r *=\left[\frac{2 \nu E_{0}\left(\gamma_{2}^{2}-1\right)}{\sigma_{\nu} \rho_{1} C^{2}}\right]^{1 / \nu}=\left[\frac{2 \nu E_{0}\left(\gamma_{2}^{2}-1\right)}{\sigma_{\nu} \gamma_{1} p_{1} M_{D}^{2}}\right]^{1 / \nu}
$$




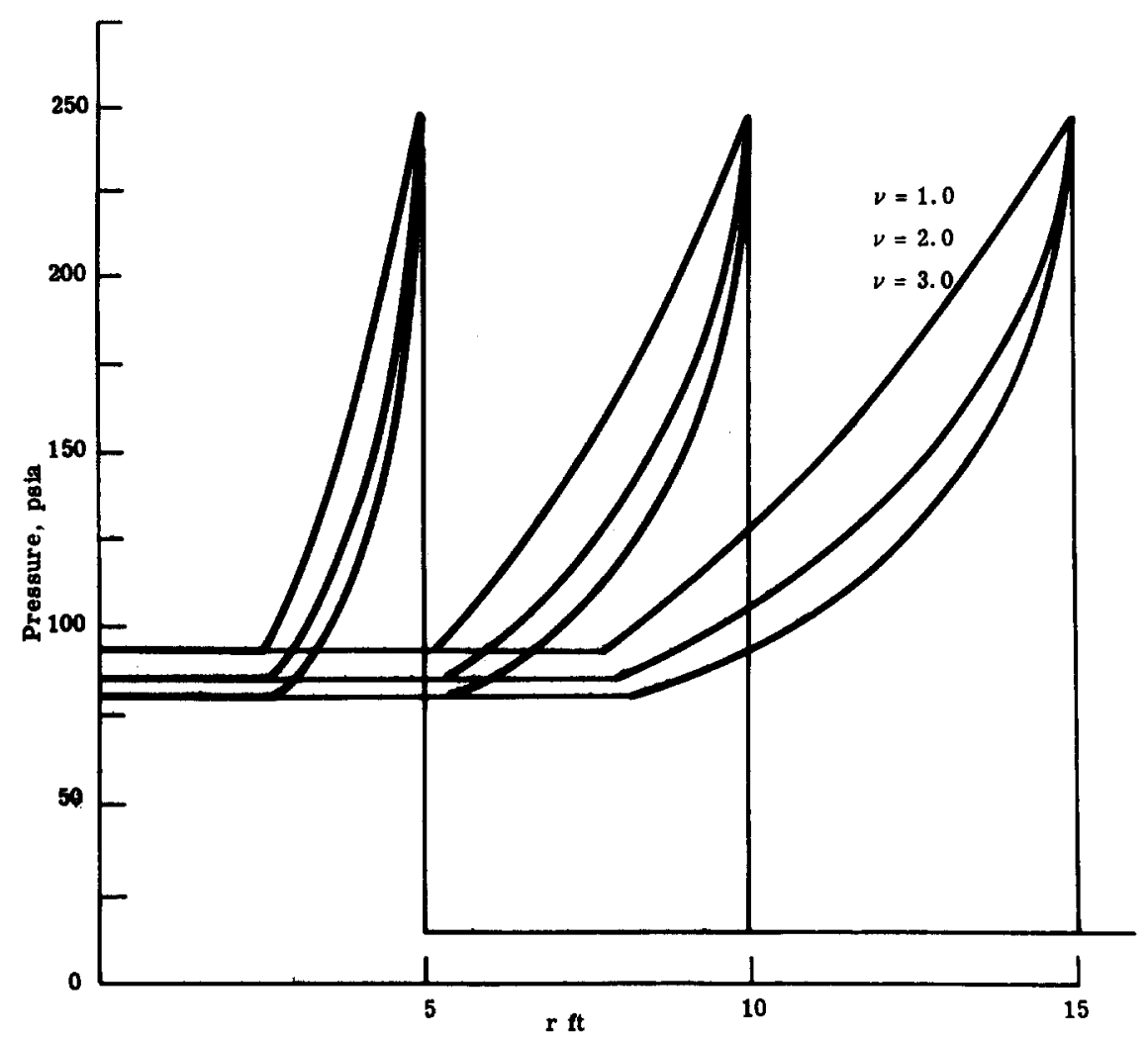

Fig. 2. Air-CH4 $\mathrm{C}-\mathrm{J}$ detonation variation of pressure.

also it is of interest to note

$$
\frac{r_{*}}{r_{0}}=\left[\frac{2 \nu\left(\gamma_{2}^{2}-1\right) k_{\nu}}{\sigma_{\nu} M_{D}^{2}}\right]^{1 / \nu}
$$

The self-similar C-J theory is readily extended to mixtures in which the fuel is in droplet form. The $\mathrm{C}-\mathrm{J}$ conditions have in this case been formulated by Williams [14] and by Nicholls et al.[15]. With the fuel in liquid form the C-J conditions are modified resulting in a slight shift in the point $\left(z_{2}, V_{2}\right)$ in the $z-V$ plane. It is generally reasonable to assume that the combustion products are in gaseous form, and then the flow behind the detonation behaves exactly as before with, however, a slight modification in the $\mathrm{C}-\mathrm{J}$ conditions.

It can be seen that with the above zeroth-order theory very little computational effort is required to determine the propagation of blast waves through combustible mixtures over a wide range of conditions. The only information required is the geometry and energy $E_{0}$ of the blast and the properties of the fuel oxidizer mixture. The theory does not provide information about the energy required for blast initiation and there is a discontinuous transition from blast to detonation properties when $r_{s}=r$. The experimental results described below show that the zeroth-order solution describes the propagation characteristics of blasts through combustible mixtures remarkably well. 


\section{Experimental research}

The present experimental investigation $[16,17]$ seeks to resolve in a controlled manner overall propagation details of blast initiated two-phase cylindrical detonation waves. Heretofore, controlled experiments have never been conducted on cylindrically or spherically propagating heterogeneous detonations wherein fuelair mixture ratio and/or drop size are controlled. The research has proceeded in two states; namely, the design and development of a satisfactory experimental facility, followed by a systematic variation of test conditions, data tabulation and analysis.

\section{Experimental facility}

A cylindrically shaped cloud was modeled which is idealized to the extent that cloud conditions (i.e., mean drop size and local mixture ratio) vary only with radius rather than with azimuth or in a direction parallel to the axis. Due to the axial symmetry of the problem and in order to minimize the magnitude of the explosion, the test chamber configuration chosen is a pie-shaped sector of a cylindrical cloud. The walls of a detonation test chamber will, in general, have an effect upon the detonation process due to heat and momentum losses to the wall. While use of a very large chamber essentially eliminates wall effects from the overall process, it is important that the chamber not be excessively large, if a test chamber is to be operated within a laboratory.

Two-phase detonation studies in constant area test chambers [18] have demonstrated that there is a relation between fuel drop size, test chamber dimensions, and the magnitude of the wall effects on the detonation process. Therefore, in the process of determining the dimensions of a test chamber that will minimize wall effects and still be of a practical size and shape, it is necessary to simultaneously consider the size of the fuel drops.

The selection of the size and distribution of the fuel drops within the test chamber must also be based on the practical limitations of the drop-producing mechanism and the fuel-to-air ratio desired. The nominal drop size was selected following an examination of the frictional and heat losses to the walls of a prospective test chamber and the reaction zone length of a detonation process. The wall losses may be taken into account through the use of Eq. (12), taken from Ref. [18], where $u_{s}$ is the actual detonation velocity, $u_{s o}$ the ideal C-J velocity, $\gamma_{3}$ the ratio of specific heats at the C-J plane, $\rho_{1}$ the fuel density, $\rho_{1}$ the oxidizer density in front of the shock wave, $C_{H}$ the heat transfer coefficient, and $r_{h}$ the hydraulic radius.

$$
u_{s} / u_{s o}=\left[1+\left(30 D / r_{h}\right) \gamma_{3}^{2}\left(\rho_{l} / \rho_{1}\right)^{1 / 2} C_{H}\right]^{-1 / 2}
$$

The product in the brackets is small compared to one, allowing $\left(u_{s o}-u_{s}\right) / u_{s o}$, the dimensionless velocity deficit to be expressed by the biomial expansion

$$
\Delta u_{s} / u_{s o}=1-\left(u_{s} / u_{s o}\right) \approx 15 D / r_{h}\left(\gamma_{3}{ }^{2}\right) C_{H}\left(\rho_{l} / \rho_{1}\right)^{1 / 2}
$$

For $\gamma_{3}=1.2, C_{H}=2.5 \times 10^{-3}$ and the appropriate densities, Eq. (13) becomes

$$
\Delta u_{s} / u_{s o} \approx 5.5 \times 10^{-5}\left(D / r_{h}\right)
$$

for $D$ in microns and $r_{h}$ in inches. 
The velocity deficit can then be expressed as a function of drop diameter and test chamber geometry, with plots similar to Fig. 3 being made for various chamber configurations. For a selected chamber geometry, the latter figure gives chamber radial distance versus drop diameter for curves of constant velocity deficit. As expected, the velocity deficit becomes very large in the chamber vertex region. It was decided that conditions should be selected that would allow the flow properties to be well established, with few wall losses, by a radial distance of 6 in. The final selection of the nominal fuel drop size and the test chamber dimensions were made on the basis of allowable limits on velocity deficit and the practical problems of drop generation. By examining a series of graphs similar to Fig. 3 and by considering that the allowable limit on the velocity deficit at a 6 in. radial distance should be about 5\%, a nominal drop size of less than $400 \mu$ was chosen.

A drop-generation technique, with important differences from that employed

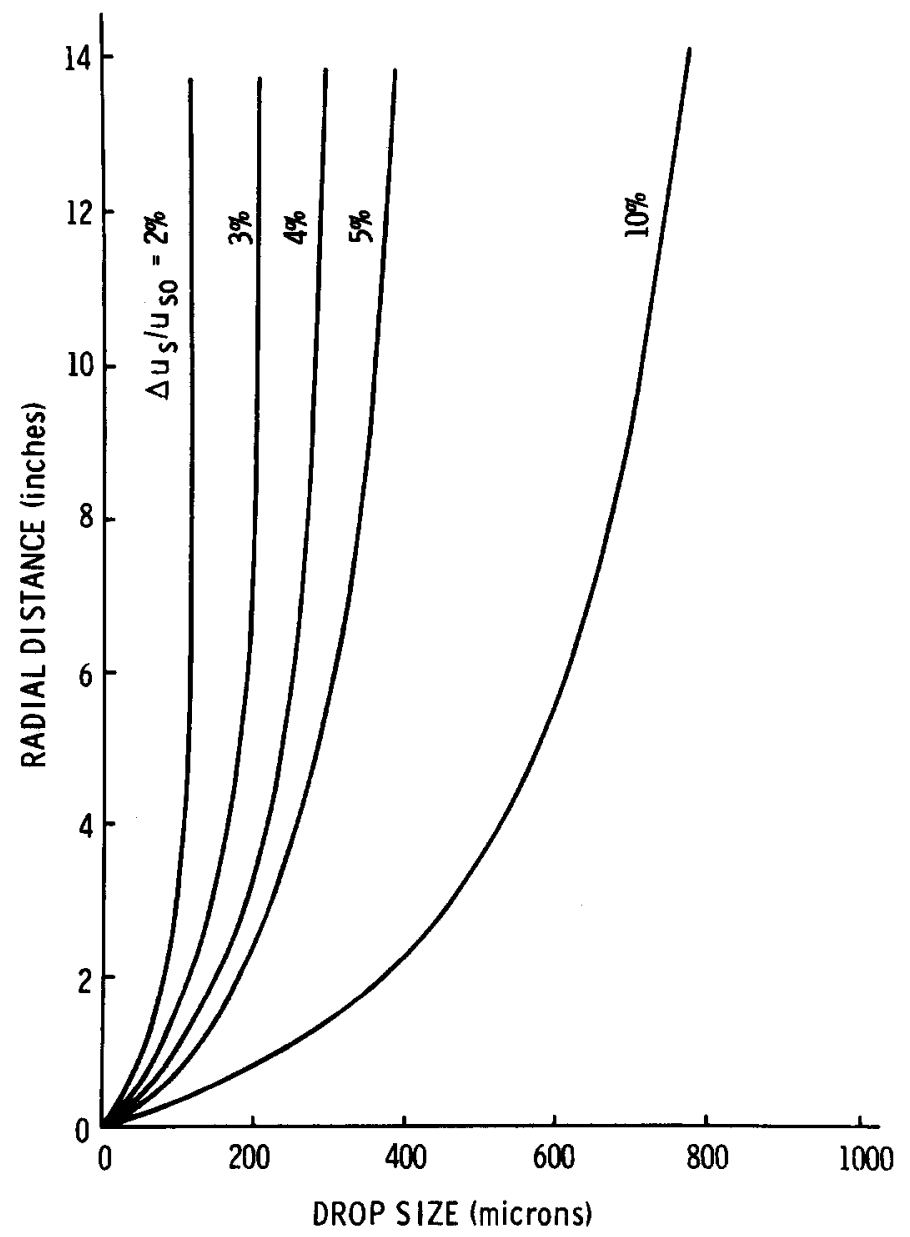

Fig. 3. Radial distance from chamber vertex plotted versus drop size for constant velocity deficit, for geometry selected. 
in earlier work [19,20,21], was developed to produce a spray of fuel drops of nearly uniform size and spacing. The decision to develop the technique was made following preliminary tests involving those already in existence, and provides for a further extension to the vibrating capillary jet methods, based essentially upon Rayleigh's analysis [22] of jet instability.

A motor-driven system was developed in order to generate stronger pressure pulses. The sinusoidal disturbances of the liquid fuel is generated by periodically interrupting the flow to the needles. By this technique, the disturbance is introduced to the fluid through rotation of a 48-tooth gear enclosed in a cylindrical case. The inlet and outlet ports are so positioned that the top land of the rotating gear simultaneously opens or closes both ports. Kerosene under pressure is fed through one port to the rotating gear. The fuel leaving the pulse generator enters the test chamber through a connected series of eleven sub-manifolds, made up of seven capillary needles each. Hence, the monodisperse spray produced in such a fashion falls vertically, orthogonal to the propagation direction of the initiating blast wave. The fuel system was thoroughly tested to determine operating conditions necessary to generate drops of uniform size and spacing. The values of drop size, and operating frequency obtained experimentally compare very favorably with those computed on the basis of Rayleigh's theory.

While the initiation of detonation in gaseous fuel-oxygen mixtures is relatively easy and many methods have been used (i.e., spark plugs, glow plugs, shock waves, and even cigars), the replacement of oxygen with air renders initiation more difficult. Nevertheless, many detonation experiments on gaseous fuel-air mixtures have been conducted. When the fuel is in liquid droplet rather than gaseous form, initiation is more difficult. In recent studies on two-phase detonation $[21,23]$, wherein the oxidizer was systematically varied from pure oxygen to oxygen-nitrogen mixtures, it was found extremely difficult to ignite the fuel when the volumetric percentage of oxygen in the oxidizer was less than about $35 \%$. In view of the above, it was deemed important to carefully consider the method of initiation in connection with the present research.

Initiation techniques considered included auxiliary shock or detonation tubes as well as solid explosives. Auxiliary shock and detonation tubes require more support equipment than the comparable solid explosive system, so that the time between experimental runs would be longer. An advantage, however, would be that these techniques can produce a sustained high pressure, high temperature, and high velocity blast directed at the drops. On the other hand, the explosive process is accompanied by a rapid decrease in these gas dynamic characteristics immediately behind the shock, which would render initiation more difficult. Nevertheless, the solid explosive technique was selected due to its simplicity, flexibility, and the ease with which the energy level could be increased. Uncertainty in the location of the energy threshold point required experimental runs of vastly differing energy releases. Blasting caps and Dupont Detasheet $\mathbf{C}$ were used in varying combinations to achieve varying amounts of readily established total energy release. The composition of Detasheet $\mathrm{C}$ is $63 \%$ Pentaerythrite Tetranitrate (PETN), 8\% Nitrocellulose (NC), and 25\% noncombustibles. The heats of combustion for PETN and NC are, respectively $4280 \mathrm{ft}-\mathrm{lb} / \mathrm{g}$ and $2640 \mathrm{ft}-\mathrm{lb} / \mathrm{g}$. 
Taking a weighted average of these, the heat of combustion for Detasheet is given as $2911 \mathrm{ft}-\mathrm{lb} / \mathrm{g}$ or $188 \mathrm{ft}-\mathrm{lb} / \mathrm{grain}$. Detasheet was exploded using Dupont blasting caps with a nominal energy release of $1110 \mathrm{ft}-\mathrm{lb}$. Hence, the total initiation energy in the tests described later is equal to the blasting cap energy added to the energy of the Detasheet used.

The detonation chamber is mounted on a Unistrut framework with the chamber centerline approximately $4 \mathrm{ft}$ above the floor. The fuel storage, motordriven fuel pressure pulse generator, fuel valving, and electrical solenoids are also mounted within the Unistrut framework. A rack cabinet immediately to one side of the detonation chamber houses most of the associated electrical and electronic equipment. Figure 4 is a schematic of the experimental apparatus.

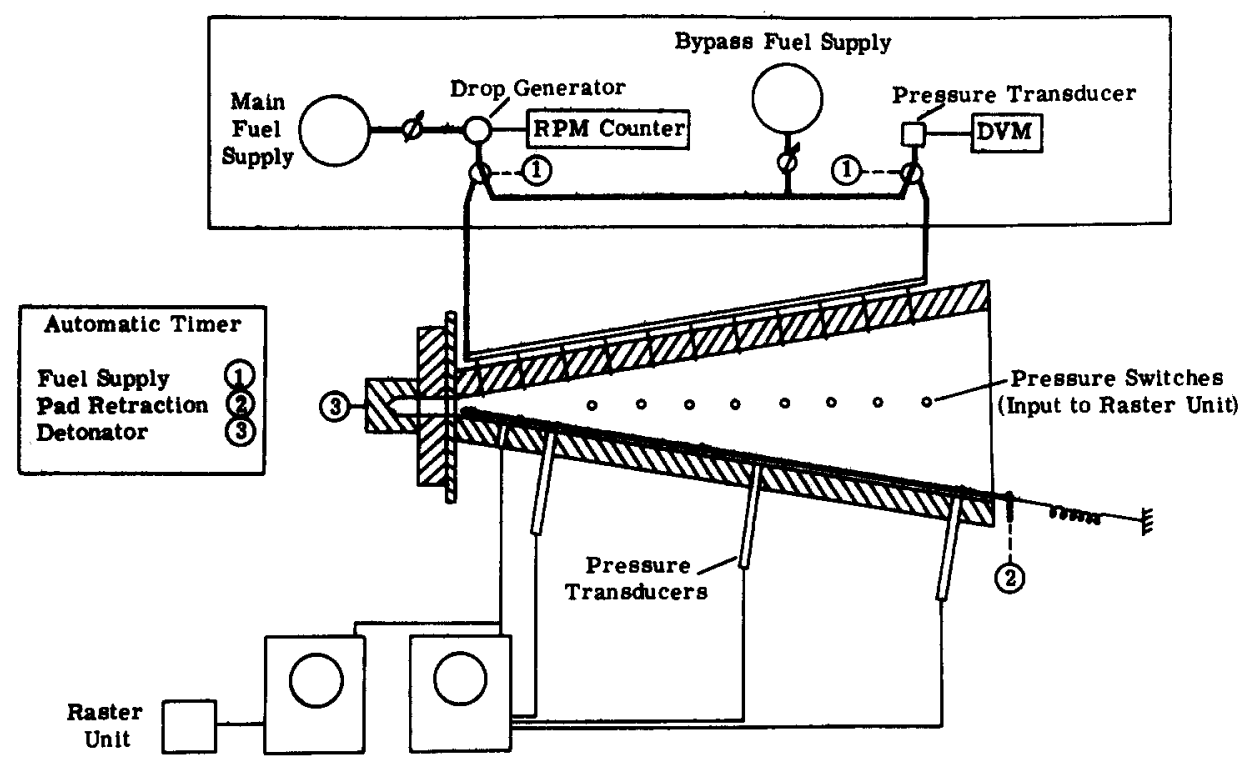

Fig. 4. Schematic of experimental apparatus.

The detonation chamber, without the breechlike explosive charge holder, is $28 \frac{3}{4}$ in. along the centerline. The top and bottom bars diverge at a $20^{\circ}$ total included angle. The inside dimensions of the chamber are $2.05 \mathrm{in}$. wide, $1 \mathrm{in}$. high at the narrow end, and 11 in. high at the open end. A breech, which holds the detonator cap and plastic explosive, is mounted to the narrow upstream end of the chamber.

The detonator tube is prepared for a run through the execution of an established sequence of steps involving final instrumentation checks, drop setup, and detonator preparation. The initiator blast wave is injected into the detonation chamber from the breech. Lead-zirconate titanate piezoelectric transducers of the type developed in earlier studies $[24,25]$, which have rise times of less than $1 \mu \mathrm{sec}$, are employed for making pressure measurements. Average wave velocities are measured over eight separate intervals using diaphragm type pressure switches of very high reliability. The output from these switches, positioned 
uniformly along the chamber side wall, is displayed by means of a raster circuit on an oscilloscope.

\section{Experimental blast wave results}

Efforts thus far have focused upon evaluation of the two limiting cases for an idealized model of a blast initiated heterogeneous detonation-the strong cylindrical blast wave and the cylindrical $\mathrm{C}-\mathrm{J}$ detonation wave.

Considerable emphasis has been placed on the attainment of good blast wave data. The reasons for this are basically threefold. First, it was an expedient way to debug much of the apparatus and instrumentation and to gain experience and confidence in the operation. Second, it was deemed important to assess the degree to which cylindrical blast waves were being generated. Third, good documentation and understanding of the blast wave data provided an experimental technique for the determination of initiation requirements for the two-phase detonation. Two basic developments followed from the blast wave experiments. An energy efficiency of the sectored chamber was determined, which relates the effective energy release with the calculated or maximum allowable energy released in the chamber. Second, a quantitative comparison of experimental blast wave data with strong blast wave theory was made, thereby yielding a behavioral estimate of wave processes in the sectored chamber.

In order to make meaningful use of the blast wave data received from these tests, it was necessary to arrive at a standard reduction technique. Once established, this technique yielded self-consistent results useful in describing the behavior of wave processes in the sectored chamber. The reduction technique finally chosen begins with a translation of the rough radius-time data from the raster scope photographs for a given energy level. This rough data is then smoothed using a least-squares polynomial regression of time data to a second-order dependency on radius. An example of this polynomial smoothing technique is given in Fig. 5, which shows a radius-time plot for the blast wave generated by $2.0 \mathrm{~g}$ of Detasheet. Displayed in the plot are the actual rough data points and the subsequent least-squares second-order polynomial fit mentioned above.

It was of interest to interpret experimental blast results in light of cylindrical blast wave theory. In order to do this, it was necessary to determine both the theoretical geometric origin and the time origin of the modeled line-source explosion. The blast center $r=0$ was assumed to be at the hypothetical apex of the chamber. Then it was necessary to determine the effective time it would take a blast wave to travel from the origin to the oscilloscope trigger station. This time is represented by $t_{0}$. As the experimentally measured time, $t^{\prime}$, is referenced from a triggering pressure switch, the time value, $t$, referred to the geometric origin, is determined by

$$
t=t^{\prime}-t_{0}
$$

where $t_{0}<0$ and is determined from data, such as shown in Fig. 5 , for each energy level. The adjusted time is then plotted against radius.

From the analysis of the blast wave data it was found that the energy release experienced in the sectored chamber was less than that predicted from the known 


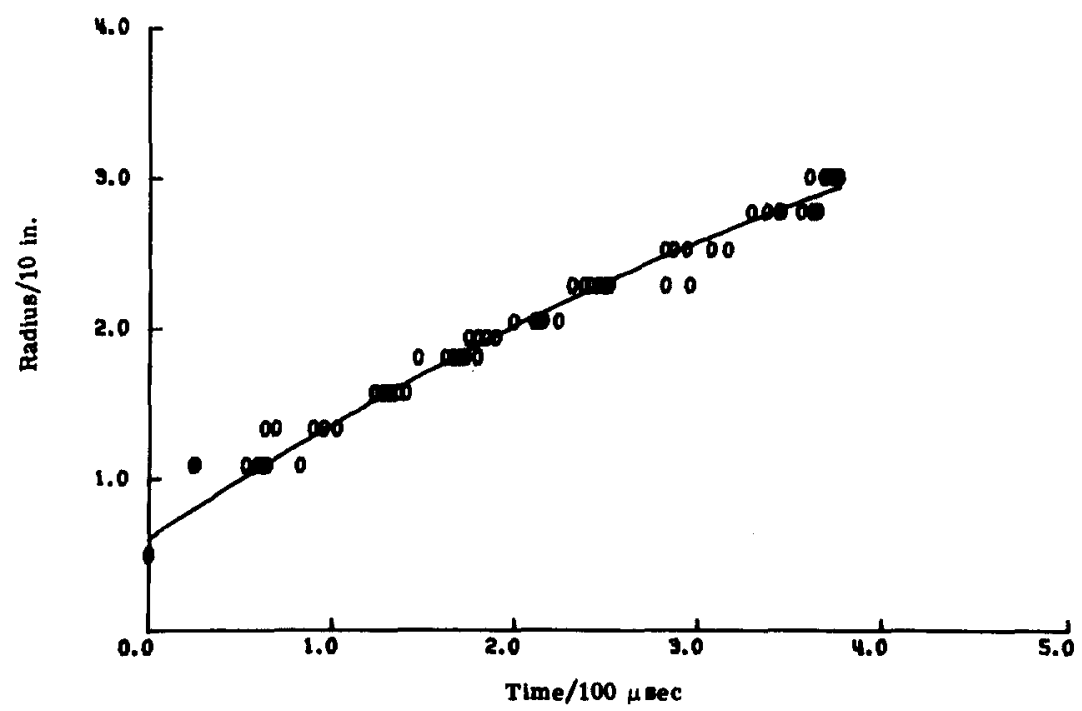

Fig. 5. Experimental blast wave data, $2.0 \mathrm{~g}$ Detasheet.

quantity of Detasheet used in the detonator. This result can be explained on the basis of the differences between the experiment and the self-similar blast wave theory with instantaneous energy release. The existence of a physically limiting chamber causes energy losses to the walls and leakage through ports in the detonator breech. The initiation energy release is not instantaneous as assumed by theory, but was actually calculated to be of the order of $10 \mu \mathrm{sec}$. Finally, the blast energy is not released uniformly along a line source.

The energy efficiencies were computed by comparing the experimental data regressed following Eq. (15) and the radius-time variation from strong cylindrical blast wave theory given in Eq. (16) (which is Eq. (3) with $\alpha=1.0$, a good approximation for $\gamma=1.4$ and $\nu=2.0$ ).

$$
\begin{gathered}
t=b_{1} r^{2} \\
t=\left(E_{0} / \rho_{1}\right)^{-1 / 2} r^{2}
\end{gathered}
$$

or

$$
t=d_{1} r^{2}
$$

Since Eqs. (15) and (16) are of the same general form, any differences in radius-time behavior between the two for a given process will be reflected in differences in the constant coefficients $b_{1}$ and $d_{1}$. An energy efficiency is now defined as

$$
\eta_{e}=E_{0_{\text {res. }}} / E_{0_{\text {cale }}}
$$

Here $E_{0_{\text {reg. }}}$ follows directly from a mathematical regression to experimental data; while $E_{0_{\text {calc. }}}$ is derived from knowledge of the detonator-Detasheet combination. Energy efficiencies have been determined for a range of energies for which experimental blast wave data was taken. Figure 6 gives the results of this computation. 




Fig. 6. Sectored chamber energy efficiency as a function of calculated energy release.

The curve displayed is a second-order polynomial regression to all the data, and was used to describe the energy efficiency in subsequent computations.

In the interest of examining how closely cylindrical blast behavior was modeled in the sectored chamber, experimental data was compared with strong blast wave theory. A quantitative comparison between experiment and theory was achieved by making use of the experimental blast data corrected by $t_{0}$ time values and the theoretical expression above, Eq. (16).

Figure 7 shows typical corrected experimental radius-time data points for a blast wave generated by $1.5 \mathrm{~g}$ of Detasheet with two curves derived from strong blast wave theory superimposed. The upper curve is strong blast behavior computed from Eq. (16) with $E_{0_{\text {calc }}}$ used as blast energy. The lower curve is the strong blast behavior corresponding to a blast energy scaled by the previously determined energy efficiency. Inasmuch as the theoretical curve involves use of an experimentally determined efficiency factor, the influence of this value on predictions was examined. Different polynomial fits to the efficiency data of Fig. 6 resulted in negligible changes in the value of $\eta_{e}$ for a given energy level. In addition, small changes in $\eta_{\boldsymbol{e}}$ were found to have little effect upon theoretical blast wave arrival times and wave velocity. It is observed from data such as shown in Fig. 7 that while the experimental wave behavior is very nearly following classical strong blast wave theory the nonidealities previously mentioned are having a modifying effect.

It is concluded that the experiments closely exhibit the desired cylindrical blast wave behavior, starting at a rather small radius.

\section{Experimental detonation wave results}

With the facility calibrated using the blast wave experiments, two phase- 


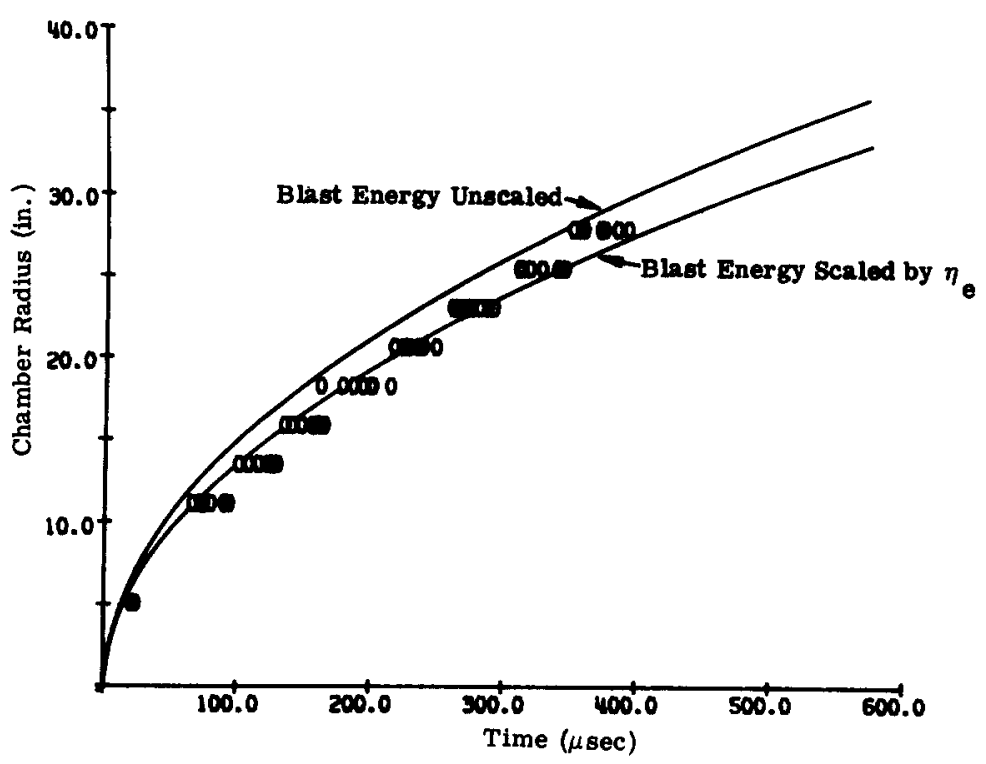

Fig. 7. Comparison of strong blast wave theory with experiment.

detonation experiments were made. Using the techniques described earlier, 77 needles were used to produce a monodisperse cloud of $380 \mu$ drops of kerosene. Two fractions of kerosene were used throughout the tests, Kerosene 1 and 2, with the latter being of a more highly refined variety. Air at atmospheric conditions was the oxidizer. The initiation energy, $E_{0}$, was systematically varied at a fixed global equivalence ratio, $\phi_{T}$, for a given fuel. To date detonation runs have been made at $\phi_{T} \approx 0.63$. Experiments with other values of $\phi_{T}$ will be made in the future.

The properties of the two kerosene fractions used are given in Table 1.

Reduction of the experimental detonation data showed it to be self-consistent and in substantiating agreement with previously reported data [16]. Figure 8 is a typical radius-time plot of experimental detonation data at varying energy levels for Kerosene 2. A corresponding plot for Kerosene 1 would show similar results. This plot demonstrates the general form of the data as well as the currently employed fourth-order polynomial fit.

Figure 9 demonstrates the current technique for obtaining experimental detonation wave propagation velocity and critical transition distance. Figure 10 is a plot of predicted two-phase detonation velocity as a function of equivalence ratio and drop size for Kerosene 2 with experimentally determined values superimposed for comparison. The predicted theoretical two-phase velocity was determined by modifying the equivalent gas phase velocity by a mean velocity deficit. The mean or average velocity deficits used in the determination of kerosene spray velocity were computed to be $1.44,2.88$, and $4.32 \%$ for 200,400 , and $600 \mu$ drop sizes, respectively. Theoretical and experimental values of detonation velocity and transition distance for Kerosene 1 and 2 are compared in Tables 2 and 3, respectively. A cursory examination of current data suggests that deviations of 
Table 1. Comparison of physical properties.

\begin{tabular}{|c|c|c|}
\hline & Kerosene 1 & Kerosene 2 \\
\hline $\begin{array}{l}\text { Density } \\
\quad\left(\text { slugs } / \mathrm{ft}^{3}\right)\end{array}$ & 1.5281 & 1.4464 \\
\hline $\begin{array}{l}\text { Surface tension } \\
(\mathrm{lb} / \mathrm{ft})\end{array}$ & $2.023 \times 10^{-3}$ & $2.059 \times 10^{-3}$ \\
\hline $\begin{array}{l}\text { Viscosity } \\
\quad\left(\mathrm{lb}-\mathrm{sec} / \mathrm{ft}^{2}\right)\end{array}$ & $5.307 \times 10^{-5}$ & $5.348 \times 10^{-5}$ \\
\hline Molecular weight & 177.06 & 206.8 \\
\hline Percent C & 86.25 & 85.44 \\
\hline Percent $\mathrm{H}$ & 13.75 & 14.56 \\
\hline $\begin{array}{l}\text { Heat of combustion } \\
\text { (cal/g) }\end{array}$ & 11,220 & 11,310 \\
\hline $\begin{array}{l}\text { Heat of formation } \\
\text { (cal/g-mole) }\end{array}$ & $-68,770$ & $-60,450$ \\
\hline $\begin{array}{l}\text { Stoichiometric } \\
\text { Equivalence ratio } \\
\left(\mathrm{O}_{2} \text { basis }\right)\end{array}$ & 0.292 & 0.292 \\
\hline
\end{tabular}

experimental velocities and transition distances from predicted values increase as the blast energy is decreased toward the ignition threshold.

Figure 11 displays in a more transparent fashion the fundamental wave behavior of a Kerosene 2-Air detonation as a function of chamber radial distance and blast initiating energy. The continuous and expected decay patterns of the

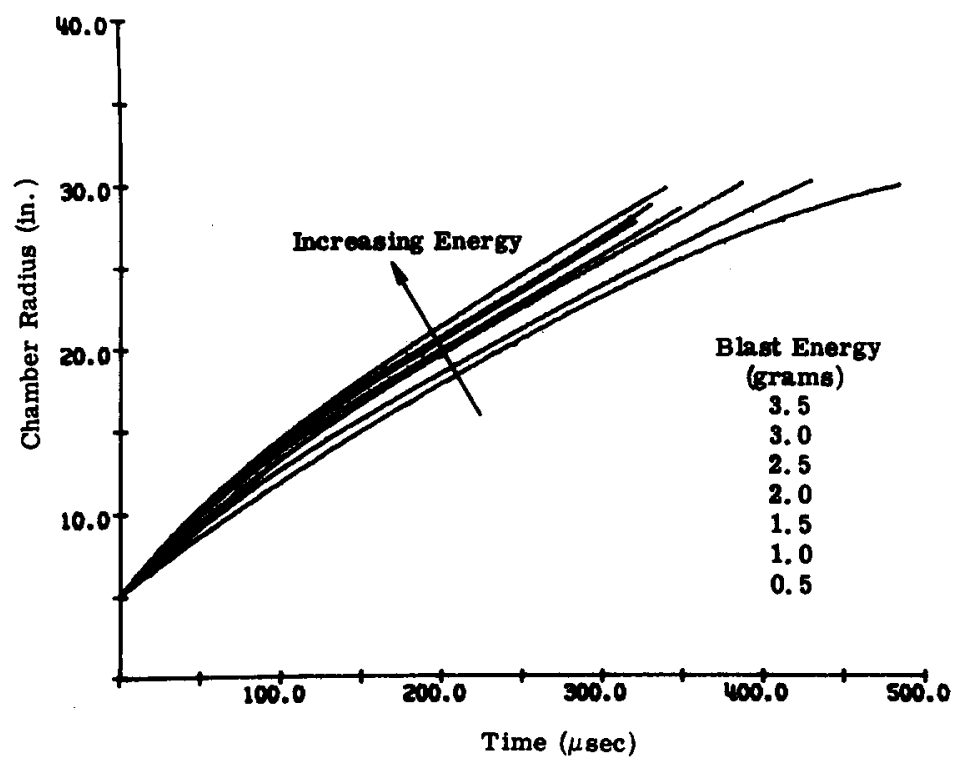

Fig. 8. Experimental Kerosene 2-Air radius-time behavior as a function of initiation energy in grams of Detasheet. 




Fig. 9. Experimental data reduction technique. (Kerosene 2-Air, $2.5 \mathrm{~g}$ Detasheet)

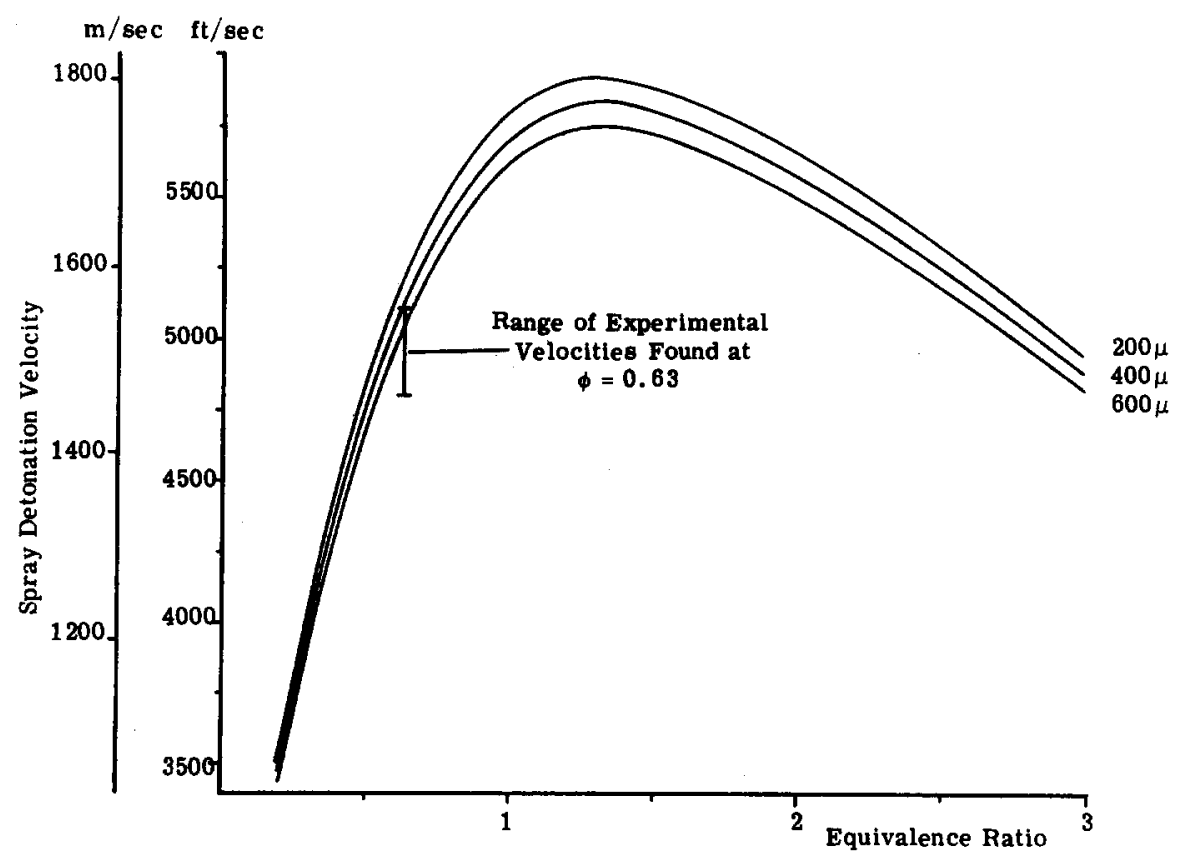

Fig. 10. Spray detonation velocity for Kerosene 2 as a function of equivalence ratio and drop size. 
Table 2. Experimental two-phase detonation results for Kerosene 1.

\begin{tabular}{ccccccc}
\hline $\begin{array}{c}\text { Explosive } \\
\text { Charge } \\
\text { (grams) }\end{array}$ & $\begin{array}{c}\text { Detonation } \\
\text { Velocity } \\
\text { (ft/sec) }\end{array}$ & $\begin{array}{c}\text { Detonation } \\
\text { Mach No. }\end{array}$ & $\begin{array}{c}\text { Percent } \\
\text { Difference }\end{array}$ & $\begin{array}{c}r_{* \circ p} \\
\text { (in.) }\end{array}$ & $\begin{array}{c}r_{* \mathrm{hoo}^{2}} \\
\text { (in.) }\end{array}$ & $\begin{array}{c}\text { Percent } \\
\text { Difference }\end{array}$ \\
\hline 2.5 & 4625 & 4.07 & 9.5 & 18.5 & 18.44 & -0.33 \\
1.5 & 4400 & 3.87 & 13.9 & 17.3 & 16.36 & -5.74 \\
\hline
\end{tabular}

'Based upon theoretical two-phase detonation velocity of $5110 \mathrm{ft} / \mathrm{sec}$.

${ }^{2}$ Computed using energy adjusted by energy efficiency factors.

curves up to the transition radius are clearly noticeable, while the slope of the curves beyond the transition radius, for sufficiently high blast energy, is constant if low-frequency polynomial generated oscillations are ignored. Thus a constant C-J detonation velocity is predicted for energies above the critical threshold, while a decaying wave velocity is predicted for sub-threshold energies. The obtained experimental detonation velocities compare to within $5 \%$ of the predicted two-phase detonation velocities. A similar plot of Kerosene 1 data shows nearly identical trends.

The general form of the experimental Mach number-radius curves of Fig. 11 then shows that the wave is initially dominated by a strong cylindrical blast wave to an energy dependent critical distance. At this critical point an abrupt transition to a constant velocity $\mathrm{C}-\mathrm{J}$ detonation wave occurs. A slight dependence of the final detonation velocity upon the initiation energy appears in these curves. At this time, however, such a dependence can only be conjecture in view of the experimental uncertainty.

Plotted in Fig. 12 are two curves of predicted critical radius and experimentally determined transition radii as functions of calculated blast wave energy. The upper curve is the critical radius computed as a function of energy from Eq. (1) with $\nu=2$, where $100 \%$ of $E_{0}$ calculated is used. The lower curve is the prediction when $E_{0}$ is scaled by the blast wave energy efficiency discussed earlier. The results are most striking, since a remarkable agreement occurs between an analytically determined critical radius and experimental values of transition radius. The

Table 3. Experimental two-phase detonation results for Kerosene 2.

\begin{tabular}{ccccccc}
\hline $\begin{array}{c}\text { Explosive } \\
\text { Charge } \\
\text { (grams) }\end{array}$ & $\begin{array}{c}\text { Detonation } \\
\text { Velocity } \\
\text { (ft/sec) }\end{array}$ & $\begin{array}{c}\text { Detonation } \\
\text { Mach No. }\end{array}$ & $\begin{array}{c}\text { Percent } \\
\text { Difference }^{1}\end{array}$ & $\begin{array}{c}r_{* \text { exp }} \\
\text { (in.) }\end{array}$ & $\begin{array}{c}r \text { theo }^{2} \\
\text { (in.) }\end{array}$ & $\begin{array}{c}\text { Percent } \\
\text { Difference }\end{array}$ \\
\hline 3.5 & 5106 & 4.50 & 0.30 & 20.3 & 20.3 & 0.0 \\
3.0 & 5090 & 4.48 & 0.60 & 19.2 & 19.31 & 0.57 \\
2.5 & 4900 & 4.32 & 4.30 & 18.2 & 18.44 & 1.30 \\
2.0 & 4800 & 4.23 & 6.30 & 17.2 & 17.51 & 1.77 \\
1.5 & 4800 & 4.23 & 6.30 & 15.8 & 16.41 & 3.72 \\
\hline
\end{tabular}

${ }^{1}$ Based upon theoretical two-phase detonation velocity of $5120 \mathrm{ft} / \mathrm{sec}$.

${ }^{2}$ Computed using energy adjusted by energy efficiency factors. 


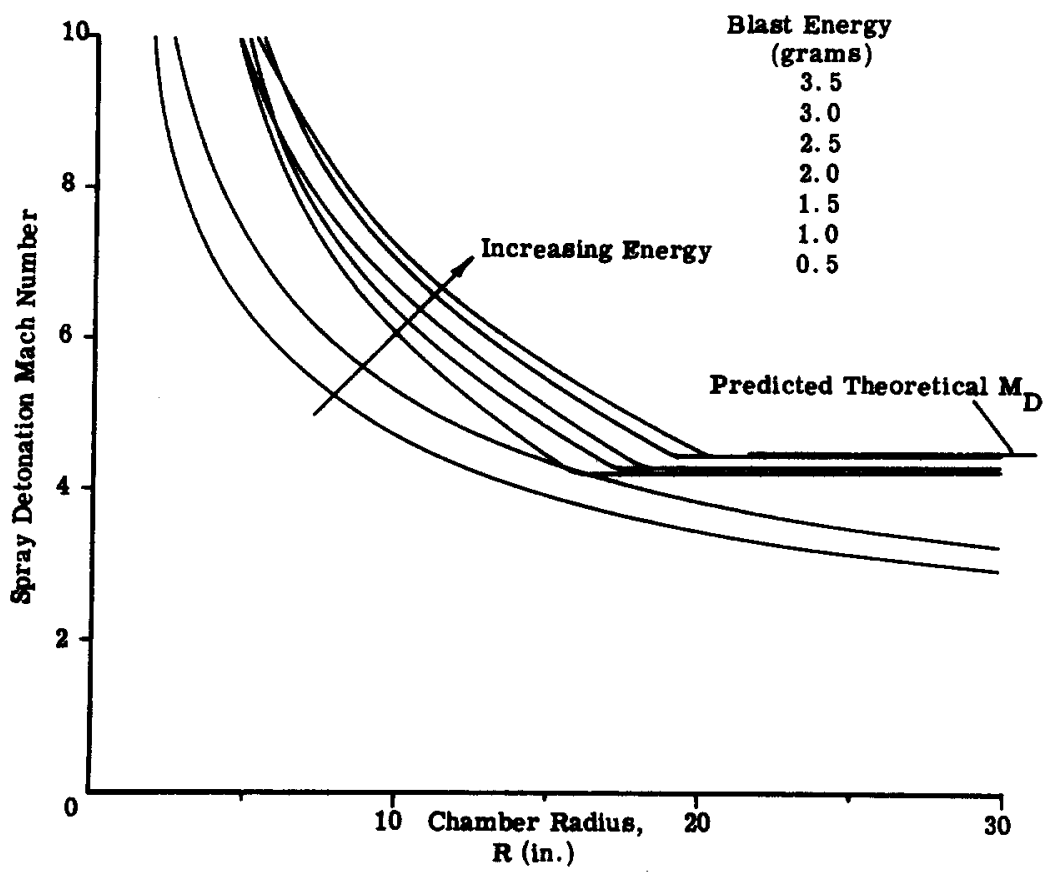

Fig. 11. Experimental two-phase detonation Mach number as a function of initiation energy and chamber radius for Kerosene $2(\phi=0.63)$.

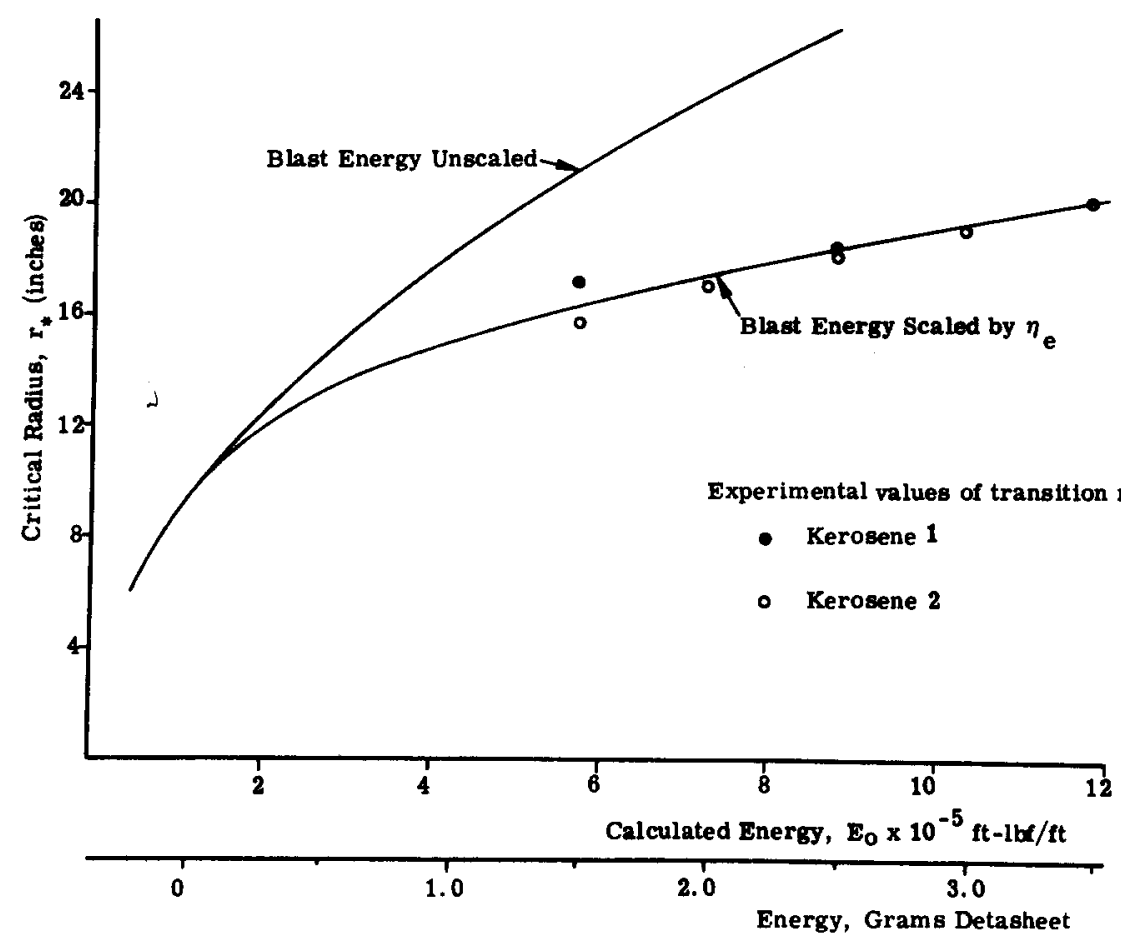

Fig. 12. Critical ignition distance as a function of energy for Kerosene 1 and 2. 
same experimentally determined value of $P_{3} / P_{1}=12.55$ was used to compute $Q$ for both kerosene fractions, thus making possible the representation of experimental transition radius values on the same plot.

\section{Conclusions}

Controlled experiments on cylindrical blast waves were performed and the associated data reduction techniques discussed. The results are interpreted to yield a calibration on the effective energy release of the initiating charge for the sectored detonation chamber.

Two-phase cylindrical detonation experiments were also conducted using two varieties of kerosene. The results indicate that at small radius blast wave behavior predominated whereas at larger radius a constant velocity detonation was realized when the initiation energy was sufficiently high. The experimentally determined transition radius between the two types of behavior agree very well with theoretical values computed using the zeroth-order solution described in Section 2 above.

Acknowledgment -This work was supported by the Air Force Armament Laboratory, Eglin Air Force Base, under Contract F08635-71-C-0083.

\section{References}

1. Lee, J. H., Gasdynamics of Detonations, Astronautica Acta 17, 455-466 (1972).

2. Chernyi, G. G., Korobeinikov, V. P., Levin, V. A. and Medvedev, S. A., One-Dimensional Unsteady Motion of Combustible Gas Mixtures Associated with Detonation Waves, Astronautica Acta 15, 259-266 (1970).

3. Korobeinikov, V. P., Gas Dynamics of Explosions, Annual Review of Fluid Mechanics 3, 317-346 (1971).

4. Korobeinikov, V. P., The Propagation of Point Explosions in a Detonating Gas, Astronautica Acta 14, 411-419 (1969).

5. Levin, V. A. and Chernyi, G. G., Asymptotic Laws of Behavior of Detonation Waves, PMM 31, 393-405 (1967).

6. Sedov, L. I., Similarity and Dimensional Methods in Mechanics, Academic Press, New York (1959).

7. Taylor, G. I., The Dynamics of the Combustion Products Behind Plane and Spherical Detonation Fronts in Explosives, Proc. R. Soc. A200, 235-247 (1950).

8. Korobeinikov, V. P., Levin, V. A., Markov, V. V. and Chernyi, G. G., Propagation of Blast Waves in a Combustible Gas, Astronautica Acta 17, 529-537 (1972).

9. Lehr, H. F., Experiments on Shock Induced Combustion, Astronautica Acta 17, 589-597 (1972).

10. Bach, G. G., Knystautas, R. and Lee, J. H., Initiation Criteria for Diverging Gaseous Detonations 13th Symp. (Inter.) on Comb., pp. 1097-1110, The Combustion Institute, Pittsburgh, Pa. (1971).

11. Strehlow, R. A., Gas Phase Detonations: Recent Developments, Combustion and Flame 12 (1968).

12. Brode, H. L., Glass, I. I. and Oppenheim, A. K., Gas Dynamics of Explosions Today, Shock Tube Research, Proc. of the Eighth Intl. Shock Tube Symp., Stoller, J. L., Gaydon, A. D. and Owen, P. R., (eds.) Imperial College, London, (July 5-8, 1971).

13. Gordon, S. and McBride, B., Computer Program for Calculation of Complex Chemical Equilibrium Compositions, Rocket Performance, Incident and Reflected Shocks, and Chapman-Jouguet Detonations, NASA SP 273 (1971).

14. Williams, F. A., Progress in Spray-Combustion Analysis, 8th (Inter.) Symp. on Comb., Williams and Wilkins, Baltimore, Md. (1962). 
15. Nicholls, J. A., Dabora, E. K. and Ragland, K. W., A Study of Two-Phase Detonation as it Relates to Rocket Motor Combustion Instability, NASA CR 272 (1965).

16. Nicholls, J. A., Fry, R. S., Glass, D. R., Sichel, M., Vander Schaff, J. and Sternstein, A. J., Fundamental Aspects of Unconfined Explosions, Technical Report, AFATL-TR- 72-49 (March 1972).

17. Nicholls, J. A., Sichel, M., Fry, R. S., Hu, C., Glass, D. R., DeSaro, R. and Kearney, K., Fundamental Aspects of Unconfined Explosions, Technical Report, AFATL-TR (March 1973).

18. Dabora, E. K., Ragland, K. W. and Nichols, J. A., Drop Size Effects in Spray Detonations, 12th Symp. (Inter.) on Comb., The Combustion Institute, Pittsburgh, Pa. (1969).

19. Dabora, E. K., Ragland, K. W. and Nicholls, J. A., A Study of Heterogeneous Detonations, Astronautica Acta 12, (1), 9-16 (1966).

20. Dabora, E. K., Production of Monodisperse Sprays, The Review of Scientific Instruments 8(4), $1648-49$ (1967).

21. Pierce, T., An Experimental and Theoretical Study of the Structure of Two-Phase Detonation in Sprays, Ph. D. Thesis, The University of Michigan (1972).

22. Rayleigh, J. W. S., Proceedings of London Mathematical Society 10, 4-18 (1878).

23. Kauffman, C. W. and Nicholls, J. A., Shock Wave Ignition of Liquid Fuel Drops, ALAA 8th Aerospace Sciences Meeting, New York, and AIAA J. (May 5 and 9, 1971).

24. Ragland, K. W. and Cullen, R. E., Piezoelectric Pressure Transducer with Acoustic Absorbing Rod, Review of Scientific Instruments 38, 740-742 (1967).

25. Rao, C. S. R., Theoretical and Experimental Study of Film Detonation, Ph. D. Thesis, The University of Michigan (1971). 